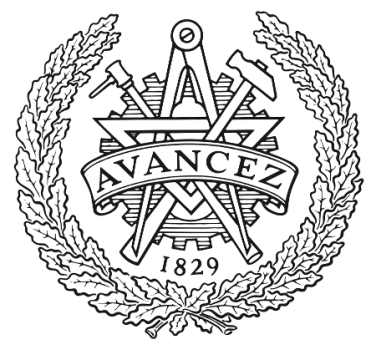

CHALMERS

UNIVERSITY OF TECHNOLOGY

\title{
Strong Plasmon-Exciton Coupling with Directional Absorption Features in Optically Thin Hybrid Nanohole Metasurfaces
}

Downloaded from: https://research.chalmers.se, 2023-04-26 13:00 UTC

Citation for the original published paper (version of record):

Kang, E., Chen, S., Sardar, S. et al (2018). Strong Plasmon-Exciton Coupling with Directional Absorption Features in Optically Thin Hybrid

Nanohole Metasurfaces. ACS Photonics, 5(10): 4046-4064.

http://dx.doi.org/10.1021/acsphotonics.8b00679

N.B. When citing this work, cite the original published paper. 


\title{
Strong Plasmon-Exciton Coupling with Directional Absorption Features in Optically Thin Hybrid Nanohole Metasurfaces
}

\author{
Evan S. H. Kang, ${ }^{\dagger}$ Shangzhi Chen, ${ }^{\dagger}$ Samim Sardar, ${ }^{\dagger}$ Daniel Tordera, ${ }^{\dagger}$ Nerijus Armakavicius, ${ }^{\ddagger}$ \\ Vanya Darakchieva, ${ }^{\ddagger}$ Timur Shegai, ${ }^{\S \odot}$ and Magnus P. Jonsson, ${ }^{*}+\odot$ \\ ${ }^{\dagger}$ Laboratory of Organic Electronics, Linköping University, SE-601 74 Norrköping, Sweden \\ ${ }^{*}$ Terahertz Materials Analysis Center, Department of Physics, Chemistry and Biology, Linköping University, SE-581 83 Linköping, \\ Sweden \\ ${ }^{\S}$ Department of Physics, Chalmers University of Technology, SE-412 96 Göteborg, Sweden
}

\section{Supporting Information}

ABSTRACT: Plasmons and excitons can interact to form new hybridized light-matter states, with a multitude of potential applications including optical logic circuits and single-photon switches. Here, we report the first observation of strong coupling based on optically thin plasmonic nanohole films. The absorptive plasmon resonances of these nanohole films lead to suppressed transmission and Fano-shaped extinction peaks. We prepared silver nanohole films by colloidal lithography, which enables large-scale fabrication of nanoholes distributed in a short-range order. When coated with J-aggregate molecules, both extinction and absorption spectra show clear formation of two separated

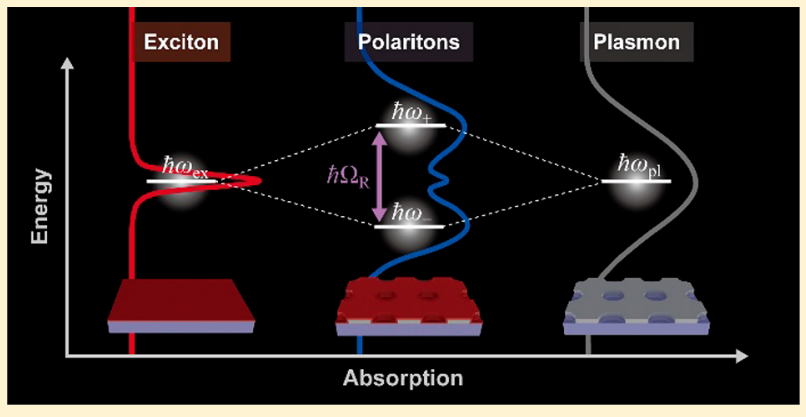
polariton resonances, with vacuum Rabi splitting on the order of $300 \mathrm{meV}$ determined from anticrossing experiments. In accordance with strong coupling theory, the splitting magnitude increases linearly with the square root of molecular concentration. The extinction peak positions are blue-shifted from the absorption polariton positions, as explained by additional Fano interference between the hybridized states and the metal film. This highlights that absorption measurements are important not only to prove strong coupling but also to correctly determine hybridized polariton positions and splitting magnitudes in hybrid plasmonic nanohole systems. The polariton absorption peaks also show strong dependence on illumination direction, as found related to inherent directionality of the plasmonic nanohole metasurface and differences in light interaction with nonhybridized molecules. Importantly, optical simulations could successfully reproduce the experimental results and all coupling features. Furthermore, simulated spatial distribution of the absorption provides additional evidence of strong coupling in the hybrid nanohole system. The work paves the way toward strong coupling applications based on optically thin nanohole systems, as further promoted by the scalable fabrication.

KEYWORDS: strong coupling, directional absorption, plasmonics, nanoholes, metasurfaces, J-aggregates, Fano interferences, polaritons

$\mathrm{T}$ he possibility to couple matter and light to form hybridized light-matter states has initiated an emerging route for creating advanced material systems with novel functionalities. ${ }^{1-5}$ This way of "dressing" molecules with light has the potential to enable concepts such as low-threshold lasers, ${ }^{6}$ single-photon switches, ${ }^{7}$ and systems with controlled chemical reactivity $^{8-11}$ or enhanced conductivity. ${ }^{12,13}$ The concept of hybridization is based on interaction between molecular absorbers and optical resonators with matching energy levels. If this interaction is sufficiently strong, the energy levels of the individual components split into several new joint energy states, in analogy to atoms adopting hybridized energy states when forming molecules. ${ }^{3}$ Hybridization occurs if the coherent energy exchange between the molecules and the optical resonator is faster than other decay channels, which defines the strong coupling regime. The strength of the interaction is manifested by the so-called vacuum Rabi splitting $\left(\hbar \Omega_{R}\right)$ governed by $\hbar \Omega_{R}=d E_{0}$, where $d$ is the transition dipole moment of the molecules and $E_{0}$ is the vacuum electric field confined by the resonator mode volume. Large energy splitting is therefore favored by a combination of molecules with large dipole moment and optical resonators with small mode volume. Plasmonic metasurfaces based on metallic optical nanoantennas have received significant attention for use as optical resonators for strong coupling studies. The plasmonic nanoantennas convert incident light to collective charge oscillations (i.e., plasmons) in the metal, resulting in strongly enhanced optical fields in small volumes. Their ultrasmall mode volume makes them particularly suitable for achieving strong coupling, ${ }^{3}$ as recently demonstrated down to the singlemolecule level at room temperature. ${ }^{14}$ Various types of

Received: May 20, 2018

Published: September 11, 2018 
plasmonic systems have been explored for strong coupling, including metal films, ${ }^{15-17}$ nanospheres, ${ }^{18}$ nanoprisms, ${ }^{19}$ bowtie nanoantennas, ${ }^{20}$ and nanoparticle arrays supporting surface lattice resonances. ${ }^{21-24}$

Among the large variety of plasmonic metasurfaces, optically thin (tens of nanometers) metal films perforated with nanoholes provide intriguing properties. ${ }^{25-27}$ Not least, they enable novel applications based on their ability to act as electrodes or fluidic nanochannels in addition to functioning as plasmonic surfaces. ${ }^{28-31}$ For optically thick nanohole films, plasmonic excitation leads to extraordinary optical transmission peaks. ${ }^{32,33}$ By contrast, plasmonic excitation in optically thin nanohole films can instead lead to suppressed transmission and a peak-dip pair in the extinction spectrum. ${ }^{34-36}$ For films thinner than the attenuation length of the metal, the surface plasmon polariton (SPP) modes of the top and bottom metal interfaces couple to form new longrange SPP (LR-SPP) and short-range SPP (SR-SPP) resonances. Only the SR-SPP is observable for nanohole films surrounded by asymmetric media (e.g., substrate and air), ${ }^{34}$ resulting in significantly enhanced absorption in the vicinity of the resonance. ${ }^{35}$ The resulting extinction peak is typically accompanied by a dip and asymmetric line shape due to Fano interference between the resonant state and the continuum background. ${ }^{37,38}$

Owing to their delocalized plasmonic modes and electrodelike structure, strong coupling based on plasmonic nanohole metasurfaces can enable novel phenomena such as conductivity enhancement of organic semiconductors. ${ }^{12}$ However, the understanding of coupling with plasmonic nanohole films is still fragmentary. In particular, previous investigations were based on optically thick nanohole films and primarily limited to using splitting of transmission peaks as an indicator of coupling behavior. ${ }^{3,39-41}$ In this respect, we note that introducing absorbing molecules in a beam path can split a transmission peak into two peaks even in the absence of any coupling. ${ }^{1}$ Therefore, further studies of strong coupling with nanoholes would benefit from systems that enable evaluation of coupling phenomena based on splitting of extinction and absorption peaks instead of transmission peaks, as standardized for nanoparticle systems. ${ }^{2}$ Thin nanohole films are suitable in this sense, because the absorptive nature of their SR-SPP resonances results in plasmon peaks in both extinction and absorption spectra. Despite this advantage, there are to our knowledge no previous reports on strong coupling with optically thin plasmonic nanohole films.

In this paper, we demonstrate strong plasmon-exciton coupling between J-aggregate molecules and thin plasmonic silver nanohole films. We report vacuum Rabi splitting of over $300 \mathrm{meV}$ at room temperature, as determined from absorption spectra, and show that the splitting increases linearly with the square root of molecular concentration. Our anticrossing analysis shows remarkably large splitting in absorption, occasionally even larger than in extinction. We provide an explanation for this observation related to extinction peak positions being shifted by Fano interference between the hybridized polariton states and the continuum background. From this, we conclude that the absorption peak positions are more suitable measures of the hybridized polariton energy levels than the extinction peak positions. Interestingly, we also find that the polariton absorption is dependent on illumination direction (front or back illumination). We attribute this to a combination of inherent directional absorption of the plasmonic nanohole system ${ }^{31}$ and differences in how nonhybridized molecules interact with the free-space light. Such asymmetric behavior was recently predicted for other strongly coupled systems, ${ }^{42}$ but to our knowledge not previously experimentally demonstrated. Importantly, our findings and conclusions are fully supported by finite-difference timedomain (FDTD) simulations, which successfully reproduced all spectral features of the hybrid nanohole systems, including the asymmetric absorption behavior and the observed differences between absorption and extinction peak positions. In addition, the simulations provide additional evidence of strong coupling via information on the spatial distribution of the local absorption in the hybridized systems.

\section{THE CONCEPT OF STRONG COUPLING IN OPTICALLY THIN NANOHOLE METASURFACES}

Figure 1 illustrates the concept of strong coupling between an optically thin plasmonic nanohole film and an overlaying

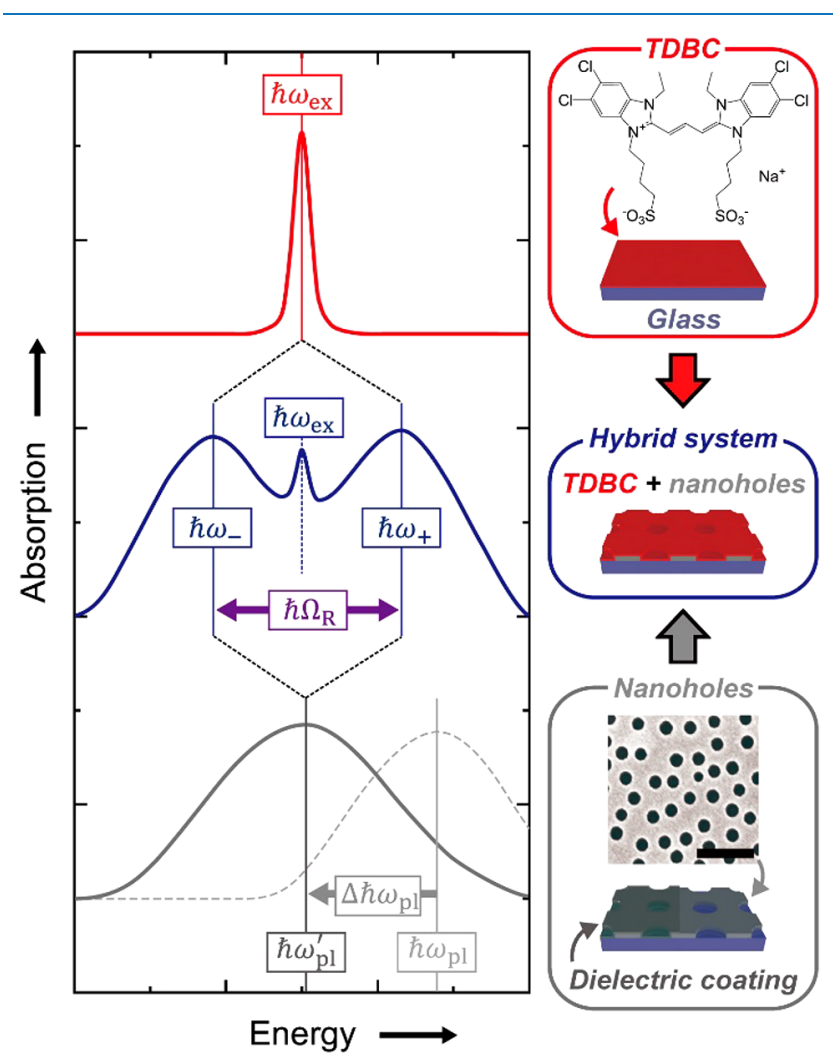

Figure 1. Schematic energy level diagram of plasmon-exciton coupling overlaid with conceptual absorption spectra for TDBC film (red solid line), TDBC-coated nanohole film (blue solid line), dielectric-coated nanohole film (gray solid line), and nanohole film (gray dashed line). Right: Sketch of the sample structures including the chemical structure of TDBC and an SEM image of an SRO nanohole film (scale bar: $500 \mathrm{~nm}$ ).

organic molecular layer. The red line illustrates molecular absorption at exciton energy $\hbar \omega_{\mathrm{ex}}$, while the gray lines correspond to the nanohole system. The gray dashed line corresponds to the bare nanohole film with plasmon resonance at $\hbar \omega_{\mathrm{p},}$ and the gray solid line accounts for the red-shift of the resonance to $\hbar \omega_{\mathrm{pl}}^{\prime}$ upon coating the metasurface with a highindex material (in analogy with how these plasmonic surfaces can be utilized for refractive index based sensing $\left.{ }^{43,44}\right)$. The middle part of the figure illustrates the hybrid system with the 


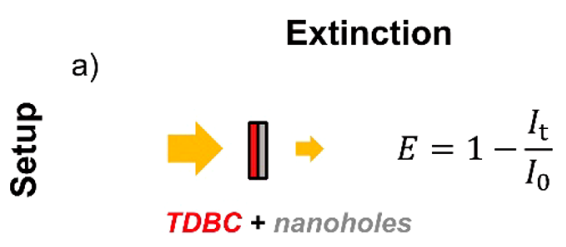

b)
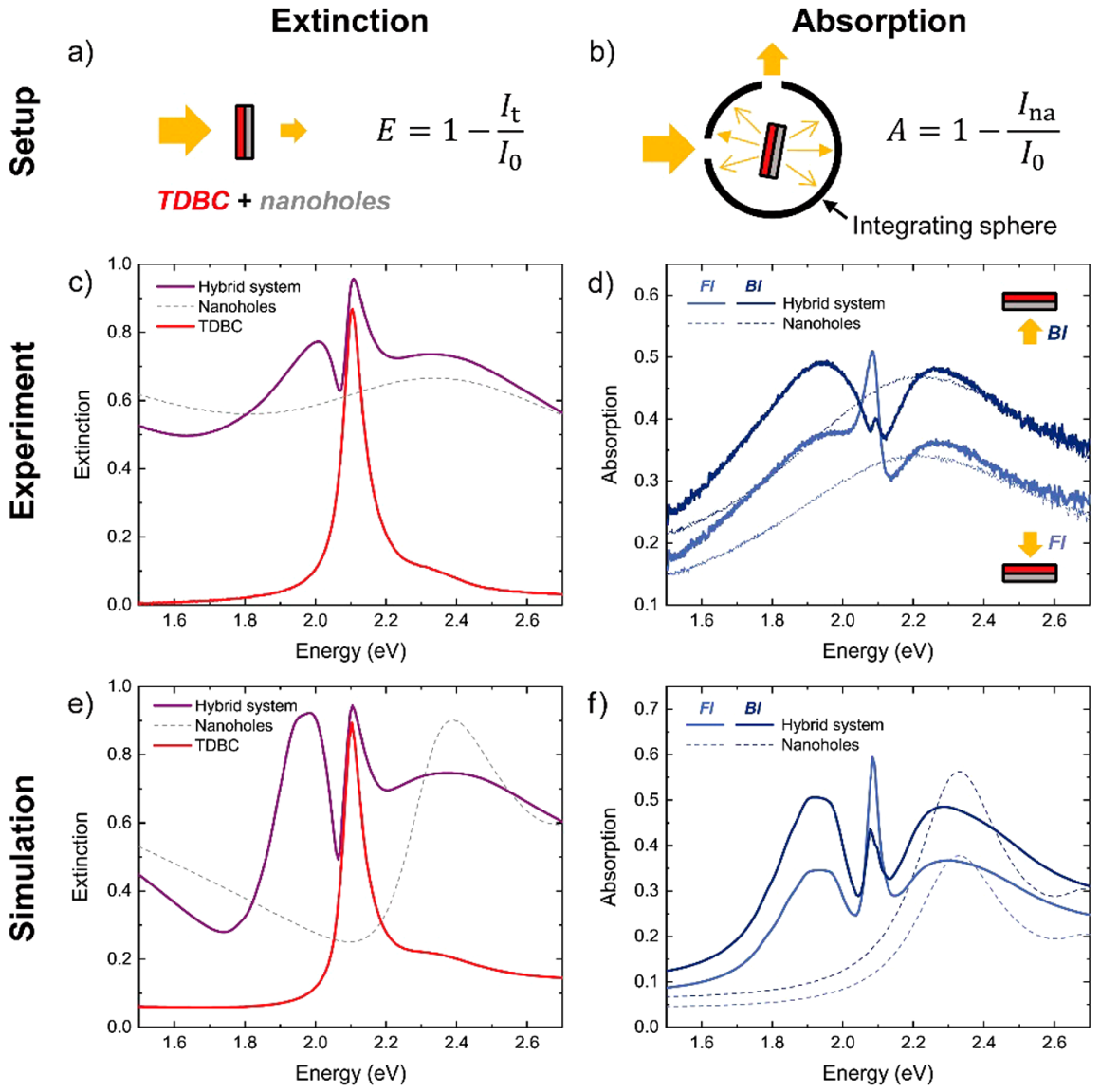

Figure 2. Schematic configurations for (a) extinction $(E)$ and (b) absorption $(A)$ measurements with $I_{\mathrm{t}}$ and $I_{\text {na }}$ corresponding to transmitted and nonabsorbed light, respectively, and $I_{0}$ corresponding to light detected with a reference sample. (c) Measured extinction spectra for TDBC on glass (red solid line), the noncoated nanohole film (gray dashed line), and the hybrid system (purple solid line). (d) Measured absorption spectra of the noncoated nanohole (dashed line) and the hybrid film (solid line) for the front (FI, light blue) and back illumination (BI, blue). Simulated (e) extinction and (f) absorption spectra corresponding to (c) and (d), respectively.

molecular layer deposited on top of the nanohole film. Hybridization can occur for this system if $\hbar \omega_{\mathrm{pl}}^{\prime}$ energetically matches $\hbar \omega_{\mathrm{ex}}$. This leads to energy level splitting and the formation of upper and lower polariton modes $\left(\hbar \omega_{+}\right.$and $\left.\hbar \omega_{-}\right)$ separated by vacuum Rabi splitting $\hbar \Omega_{\mathrm{R}}$. The conceptual absorption curve also includes a small contribution at $\hbar \omega_{\mathrm{ex}}$ representing nonhybridized molecules remaining in the system.

To experimentally investigate coupling with optically thin plasmonic nanoholes, we used colloidal lithography to prepare a $20 \mathrm{~nm}$ thin silver film perforated with $110 \mathrm{~nm}$ in diameter nanoholes on a glass substrate (see fabrication details in the Supporting Information). ${ }^{45}$ This gave nanoholes distributed over the entire sample in a short-range order (SRO), with a characteristic center-to-center distance determined to be 200 $\mathrm{nm}$ from the radial distribution function of scanning electron microscopy (SEM) images (see Figure S1). The gray dashed line in Figure $2 c$ shows the bare nanohole film's extinction spectrum with its characteristic Fano line shape (setup configuration illustrated in Figure 2a). We then prepared the hybrid plasmon-exciton system by coating the plasmonic nanohole film with a $7.5 \mathrm{~nm}$ thick layer of the J-aggregated cyanine dye TDBC (5,6-dichloro-2-[[5,6-dichloro-1-ethyl-3(4-sulfobutyl)benzimidazol-2-ylidene]propenyl]-1-ethyl-3-(4sulfobutyl)benzimidazolium hydroxide, inner salt, sodium salt). TDBC has a well-defined exciton energy level at $\hbar \omega_{\text {ex }}$ manifested as a narrow extinction peak at $2.1 \mathrm{eV}$ for the bare TDBC film on a glass substrate (red curve in Figure 2c).
The hybrid system clearly shows three distinguishable extinction peaks (purple solid line), where the two outer peaks correspond to the formation of two separated polariton resonances and the middle peak corresponds to nonhybridized molecules in regions of less strong plasmonic fields. Similar nonhybridized peaks have been reported for hybrid nanodisk arrays and ascribed to weak electric field regions between nanodisks. ${ }^{46}$

\section{STRONG COUPLING MEASURED BY ABSORPTION}

Although commonly used in the literature to report strong coupling, recent studies show that splitting in extinction (or scattering) does not alone constitute unambiguous evidence of strong coupling. ${ }^{1,3}$ The reason is that other mechanisms associated with the weak coupling regime, such as Fano resonances, can also lead to a scattering dip, thereby resulting in a splitting-like shape of the extinction spectrum even without true energy level splitting. ${ }^{18,47,48}$ To distinguish strong coupling from weak coupling mechanisms, it is therefore essential to also investigate Rabi splitting behavior in absorption. ${ }^{18,48-51}$ We used an integrating sphere to measure the absorption of the nanohole sample with and without TDBC coating (setup configuration illustrated in Figure $2 b$ ). The results are presented in Figure $2 \mathrm{~d}$ for illumination from either the front (light blue) or back (blue) of the sample. It is clear that coating the nanohole film with the TDBC layer leads 

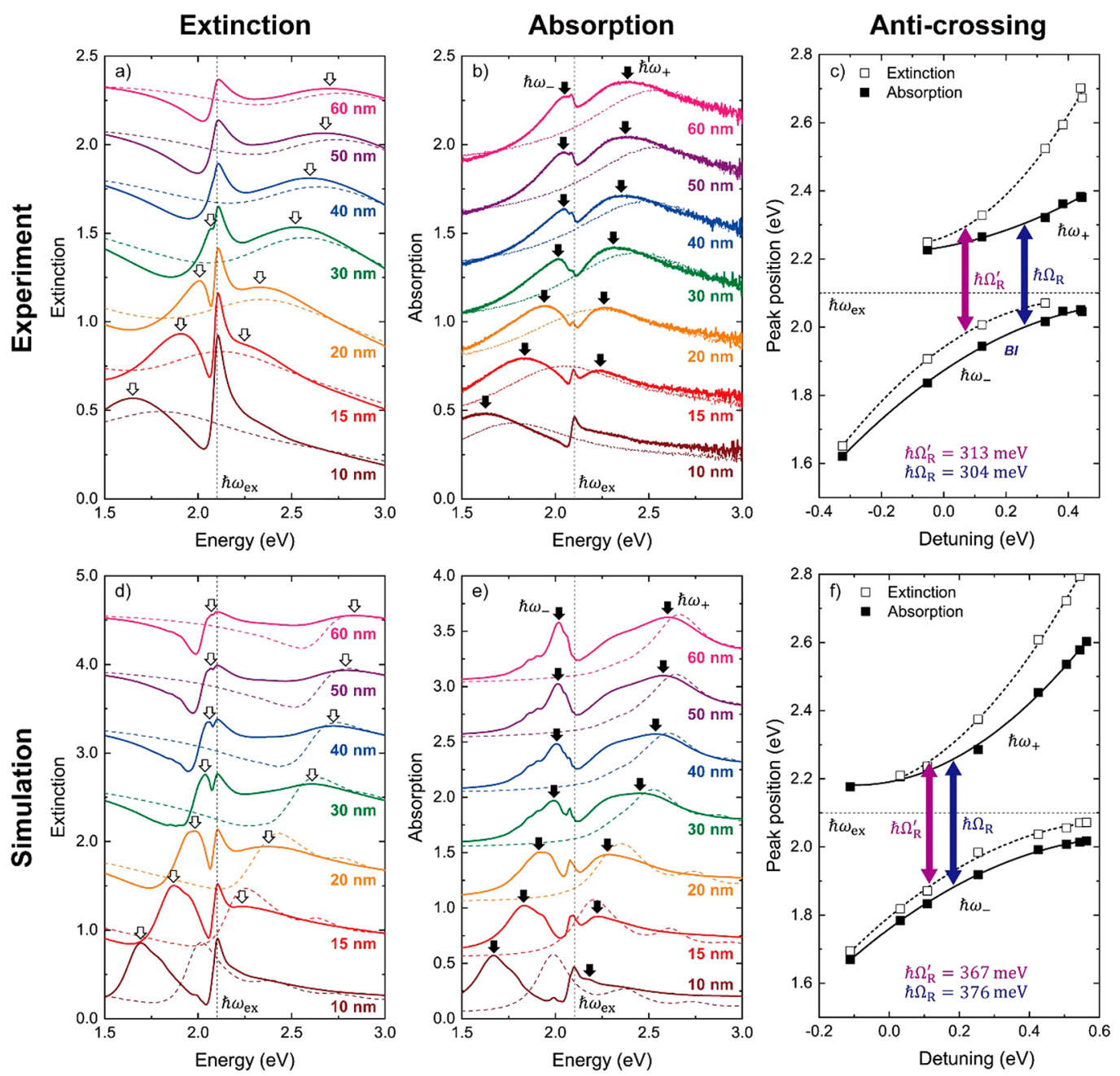

Figure 3. Measured (a) extinction and (b) absorption spectra for the bare nanohole films (dashed lines) and the TDBC-coated nanohole films (solid lines) of various thicknesses. White and black arrows designate extinction and absorption peak positions, respectively. The nonhybridized exciton energy level is indicated by the black vertical dotted line at around $2.1 \mathrm{eV}$. (c) Anticrossing plot constructed using the peak positions in (a) and (b). Minimum splitting in extinction and absorption are marked with a purple and a blue arrow, respectively. (d-f) Results calculated by FDTD simulations corresponding to (a) - (c). Spectra for (a), (b), (d), and (e) are plotted with vertical offsets for better visibility.

to splitting also in absorption, manifested as splitting of the original plasmonic absorption peaks (dashed lines) into two new hybridized polariton peaks (solid lines). This absorption splitting forms the first experimental proof of hybridization and energy level splitting in hybrid optically thin plasmonic nanohole films, and it is also the first demonstration of hybridization based on SRO nanohole films.

We performed FDTD simulations to solidify our experimental results, with the silver nanohole film represented by $110 \mathrm{~nm}$ in diameter holes distributed in a square array with $200 \mathrm{~nm}$ periodicity. ${ }^{25-27}$ For the TDBC layer, we used permittivity data obtained by ellipsometry (Figure S2) and set the thickness to $7.5 \mathrm{~nm}$ as measured by atomic force microscopy (effectively 8 at $1 \mathrm{~nm}$ mesh resolution). Notably, the resulting simulated spectra of the hybrid nanohole system successfully reproduce all essential features of the experimentally obtained extinction (Figure 2e) and absorption (Figure 2f) spectra. The narrower polariton resonances in the simulated spectra can be attributed to the sharper $\hbar \omega_{\mathrm{pl}}$ for the perfect periodic systems compared to the imperfect experimental SRO structures. It is worthwhile to note that the plasmon resonance of the bare nanohole film does not seem to match the TDBC exciton resonance in either extinction or absorption spectra, yet we observe strong and relatively symmetric splitting. As illustrated in Figure 1, this is due to the plasmon resonance being red-shifted (to $\left.\hbar \omega_{\mathrm{pl}}^{\prime}\right)$ upon coating by TDBC, enabling energy matching for the hybrid system. Indeed, FDTD simulations presented in Figure S4 demonstrate that nonabsorbing materials with a real refractive index comparable to that of TDBC can shift $\hbar \omega_{\mathrm{pl}}$ the few hundreds of $\mathrm{meV}$ needed for energy matching.

\section{DIRECTIONAL POLARITON ABSORPTION}

We will now discuss a striking feature in the hybridized absorption spectra; namely, they are strongly asymmetric and influenced by the direction of the illumination. The absorption upon illumination from the front of the sample (light blue solid line in Figure $2 \mathrm{~d}$, front illumination, FI) to a large extent resembles the extinction spectrum (Figure 2c), including a clearly pronounced absorption peak by nonhybridized molecules. On the contrary, illumination from the back side of the glass substrate (blue solid line in Figure 2d, back illumination, BI) gives only minimal absorption from nonhybridized molecules, while the two hybridized polariton peaks 
become very clear and even more pronounced than for the extinction measurements. These features are all consistent with the simulated results (Figure 2f). We attribute the more distinct polaritonic absorption peaks for BI compared to FI partly to the directional absorption behavior of the noncoated nanohole samples (dashed lines). Indeed, plasmonic nanoantennas can be designed to provide directional properties resembling those of traditional radio and television antennas, ${ }^{52-55}$ and we recently reported directional absorption for plasmonic nanohole systems similar to those used in this work. $^{31}$ To our knowledge, this is the first report experimentally demonstrating that directional optical nanoantennas or metasurfaces can be used to form strongly coupled plasmon-exciton systems with directional properties. Similar asymmetric behavior was recently theoretically predicted for a hybrid metasurface-quantum well system. ${ }^{42}$

The reduced nonhybridized absorption peak obtained in the BI geometry is primarily ascribed to less free-space light reaching nonhybridized TDBC molecules after transmission through the metal nanohole film compared to direct FI illumination. In turn, this means that BI forms a more effective probe to investigate coupling behavior between plasmons and excitons in perforated plasmonic systems, by minimizing interaction of nonhybridized molecules with direct light illumination. This interpretation agrees with simulated absorption for a TDBC-coated nonperforated silver film, which showed significantly weaker TDBC absorption for BI compared to FI (Figure S5).

Based on our observation that the hybridized absorption states are more pronounced for BI than for FI, the question arises whether the hybridization itself is stronger and occurs preferentially for plasmon modes associated with BI. If so, we would expect larger Rabi splitting for BI compared with FI. However, at least for our system, we find no noticeable differences in the magnitude of the Rabi splitting for the different illumination directions. We therefore conclude that BI amplifies the absorption signal of the plasmon and polariton resonances without disturbing the underlying physics of the coupling.

\section{ANTICROSSING AND VACUUM RABI SPLITTING}

After establishing that splitting occurs both in extinction and in absorption, we will now quantify the vacuum Rabi splitting through anticrossing analysis and discuss differences between extinction and absorption. For this, we tune $\hbar \omega_{\mathrm{pl}}^{\prime}$ around the region of $\hbar \omega_{\text {ex }}$ using nanohole films of different thicknesses. In the strong coupling regime, such tuning should lead to an anticrossing pattern with the hybridized polariton energy levels avoiding degeneration of the original resonances $\left(\hbar \omega_{\mathrm{pl}}^{\prime}\right.$ and $\left.\hbar \omega_{\mathrm{ex}}\right)$. The vacuum Rabi splitting $\left(\hbar \Omega_{\mathrm{R}}\right)$ is defined as the minimum splitting obtained at $\hbar \omega_{\mathrm{pl}}^{\prime}=\hbar \omega_{\mathrm{ex}}{ }^{56}$ Figure 3 presents our anticrossing studies for both extinction and absorption. As indicated, top and bottom panels correspond to experimental and simulated results, respectively. The dashed lines in Figure $3 \mathrm{a}$ and $\mathrm{b}$ respectively present the extinction and absorption spectra of the bare nanohole films of various thicknesses (all with a hole diameter of $110 \mathrm{~nm}$ and characteristic hole-to-hole spacing of $200 \mathrm{~nm}$ ). Consistent with previous studies, ${ }^{37,57} \hbar \omega_{\mathrm{pl}}$ red-shifts with decreasing film thickness, clearly sweeping across the position of $\hbar \omega_{\text {ex }}(\sim 2.1$ $\mathrm{eV}$ ). Coating the same samples with the TDBC layer leads to the solid curves in Figure 3. We first note that even for thicknesses farthest from energy matching (largest detuning,
10 and $60 \mathrm{~nm}$ ), the extinction spectra still show an asymmetric Fano profile around $\hbar \omega_{\mathrm{ex}}$. This is in agreement with extinction results also for nonstructured metal films coated with absorbing molecules. ${ }^{58}$ For thicknesses providing better energy matching $\left(\hbar \omega_{\mathrm{pl}}^{\prime}\right.$ closer to $\left.\hbar \omega_{\mathrm{ex}}\right)$, both absorption and extinction spectra clearly show energy level splitting and the advent of two polariton states, $\hbar \omega_{+}$and $\hbar \omega_{-}$.

Extraction of peak positions from the extinction and absorption spectra in Figure $3 \mathrm{a}$ and $\mathrm{b}$ allowed us to construct anticrossing plots, as presented in Figure 3c. Here, the peak positions are plotted as a function of detuning $\left(\hbar \omega_{\mathrm{pl}}-\hbar \omega_{\mathrm{ex}}\right)$ instead of nanohole film thickness, with $\hbar \omega_{\mathrm{pl}}$ obtained from absorption spectra of the noncoated nanohole films. The white squares indicate the extracted extinction peak positions (marked with white arrows in Figure 3a), and the black squares indicate the extracted absorption peak positions (marked with black arrows in Figure 3b; see Figure S6 for details regarding the procedure of extracting peak positions). The dashed and solid lines in Figure $3 \mathrm{c}$ are polynomial fits interpolating the extinction and absorption data, respectively. The simulated results corresponding to Figure $3 a-c$ are presented in Figure $3 \mathrm{~d}-\mathrm{f}$, with an overall good agreement.

In examining these results, we first note that both absorption and extinction form clear anticrossing patterns, with a minimum splitting at nonzero detuning due to the red-shifted plasmon resonance by the TDBC coating. The fact that minimum splitting occurs for different positions for extinction (purple double arrow) and absorption (blue double arrow) is related to the extinction positions being affected by Fano interference effects, as discussed in more detail below. Experimental values extracted for the vacuum Rabi splitting in extinction $\left(\hbar \Omega_{\mathrm{R}}^{\prime}\right)$ and absorption $\left(\hbar \Omega_{\mathrm{R}}\right)$ are both around $300 \mathrm{meV}$ (see Figure S7 for details). These values are reasonable and similar to those reported based on transmission peak splitting for periodic arrays of nanoholes in optically thick films. ${ }^{39-41}$ Interestingly, the Rabi splitting extracted from absorption measurements $(304 \mathrm{meV})$ is very similar in magnitude to the measured extinction splitting $(313 \mathrm{meV})$, and for the simulated results the absorption splitting (376 $\mathrm{meV}$ ) is even larger than the extinction splitting $(367 \mathrm{meV}$, Figure $3 \mathrm{f}$ ). This behavior is opposite that reported for other hybrid plasmon-exciton systems, where large splitting in scattering typically leads to large splitting in extinction even when absorption shows little or no splitting. ${ }^{49,59}$ Only when coupling of absorptive resonances dominates can the magnitude of the splitting in absorption and extinction be comparable, yet still not larger. This distinctive feature, as well as the difference in detuning position for absorption and extinction, can be understood from a discussion based on Fano interference effects due to the continuum background of the metal film. We stress that these Fano effects should not be confused with weak plasmon-exciton coupling between the nanohole films and the molecules. Instead, they should be considered as additional interference effects affecting the positions at which the strongly coupled hybridized modes appear in the extinction spectra. Indeed, Fano interference will blue-shift the extinction peaks from the true resonance positions. ${ }^{60}$ This can be seen in Figure $3 \mathrm{c}$ and $\mathrm{f}$, for which the extinction peak positions for all samples are located at higher energies than the corresponding absorption peak positions (also see Figure S8 for a direct comparison between the extinction and absorption spectra for a hybrid nanohole system). While both upper and lower polariton positions are 

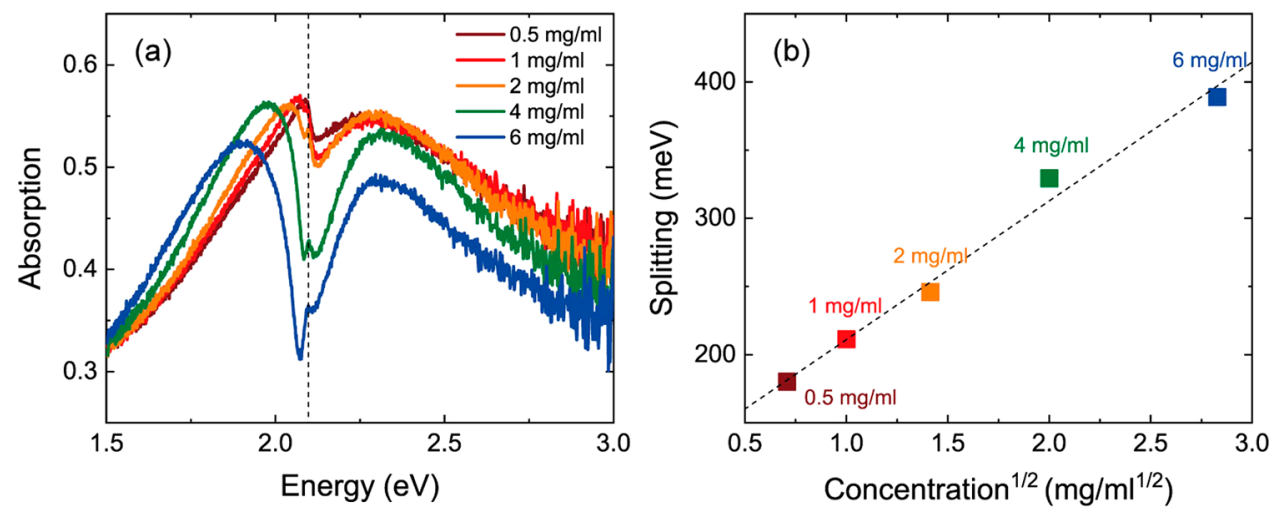

Figure 4. (a) Absorption spectra for $20 \mathrm{~nm}$ thick nanohole films coated using various TDBC solution concentrations. The vertical dashed line indicates the original exciton resonance at $\sim 2.1 \mathrm{eV}$. (b) Splitting magnitude extracted from (a) as a function of square root of TDBC concentration. The dashed line is a linear fit to the data.

blue-shifted, the shifts are of different magnitudes. This affects the apparent energy separation between the polaritons and, hence, the magnitude of the extracted extinction splitting. Furthermore, this difference in blue-shift between upper and lower extinction polariton peaks also varies with detuning (i.e., film thickness), which for our system results in underestimated extinction splitting at negative detuning and overestimation at large positive detuning (see Figure S10). This explains why the measured splitting can be lower in extinction than in absorption and also why minimum splitting occurs at lower detuning for extinction than for absorption for our system. As discussed in more detail in the Supporting Information, the variations of the blue shifts with detuning for the different polaritons may be related to secondary effects appearing upon superposition of multiple closely positioned Fano peaks (see Figures S9 and S10 and related discussion). To summarize this part, extracting polariton positions and vacuum Rabi splitting from extinction spectra can lead to erroneous results for plasmonic systems that involve Fano interferences in addition to the hybridized strong coupling. Since these Fano effects are not expected to affect the absorption spectra, ${ }^{48,61}$ we conclude that the absorption peaks are better suited for determining the polariton energy levels and vacuum Rabi splitting for our hybrid plasmonic nanohole metasurfaces, as well as for other systems with similar features.

\section{EVALUATION OF COUPLING BEHAVIOR: PROVING STRONG COUPLING}

The coupling behavior can be assessed quantitatively by comparing the Rabi splitting energy with the line widths of the individual resonances. A common criterion for the strong coupling regime is $\hbar \Omega_{\mathrm{R}}>\frac{1}{2}\left(\gamma_{\mathrm{pl}}+\gamma_{\mathrm{ex}}\right)$, where $\gamma_{\mathrm{pl}}$ and $\gamma_{\mathrm{ex}}$ are the line widths of the plasmon resonance and exciton absorption, respectively. ${ }^{2,62}$ It is not straightforward to determine $\gamma_{\mathrm{pl}}$, because the nanohole absorption spectrum (blue dashed line in Figure 2d) also contains contribution from wavelength-dependent nonresonant absorption in the metal film. Assuming a linear background absorption, we estimated the line width from a Gaussian fit to the absorption spectrum for the $20 \mathrm{~nm}$ thick nanohole film (see Figure S11a). This gives $\gamma_{\mathrm{pl}} \approx 757 \mathrm{meV}$, which with $\gamma_{\mathrm{ex}} \approx 80 \mathrm{meV}$ and $\hbar \Omega_{\mathrm{R}}=304$ $\mathrm{meV}$ brings us near the border of the strong coupling regime. Notably, this criterion should be considered merely as a rule of thumb, and hybridization has been shown to occur also for smaller splitting. ${ }^{3}$ Moreover, for our nanohole system, the extraction of the line width from the absorption spectrum was sensitive to small variations in fit parameters (e.g., wavelength range and baseline), making the evaluation less reliable. Regarding plasmon line width, it should also be noted that experimental inhomogeneity (variations in hole diameter and spacing) may result in apparent line-width broadening for ensemble measurements, thereby underestimating the resonance quality factor. ${ }^{18}$ Indeed, this is in agreement with the ideal simulated systems showing sharper plasmon resonances, although this is likely also related to differences in hole distribution (see Figure S11b for the line widths of the simulated resonances). Considering these points above and that the splitting was evaluated based on absorption spectra, the anticrossing analysis indicates that the hybrid nanohole system is in the strong coupling regime. The simulated system is clearly in the strong coupling regime, with not only sharper resonances but also slightly larger Rabi splitting than the experimental system. The latter may be related to lower damping as well as differences in mode volume or small variations in coating properties.

As additional evaluation of whether or not the hybrid plasmonic nanohole metasurfaces are in the strong coupling regime, we investigate how $\hbar \Omega_{\mathrm{R}}$ varies with TDBC concentration. Figure 4 a shows BI absorption spectra obtained for the $20 \mathrm{~nm}$ think plasmonic nanohole films coated using solutions of different concentrations of TDBC. We find that the splitting increases with increasing concentration and also that the absorption dip at $\hbar \omega_{\text {ex }}$ becomes more pronounced. Extracting splitting magnitudes from these spectra shows that $\hbar \Omega_{\mathrm{R}}$ increases linearly with the square root of TDBC concentration (Figure 4b), which forms an independent proof that the system is in the strong coupling regime. $1,39,40,63$ The same dependence was found for simulated results based on approximating the TDBC permittivity by Lorentzian functions of varying oscillator strengths (Figure S12).

\section{SPATIAL ABSORPTION DISTRIBUTION}

FDTD simulations were further implemented to obtain additional insight into the contribution to the total absorption from different regions within the hybrid nanohole systems. This method allowed us to calculate the local power absorption per unit volume $\left(P_{\mathrm{abs}}\right)$ as a function of position $(x, y, z)$ and excitation energy. By integrating $P_{\mathrm{abs}}$ over different volumes, we first used this approach to determine the absorption in the 


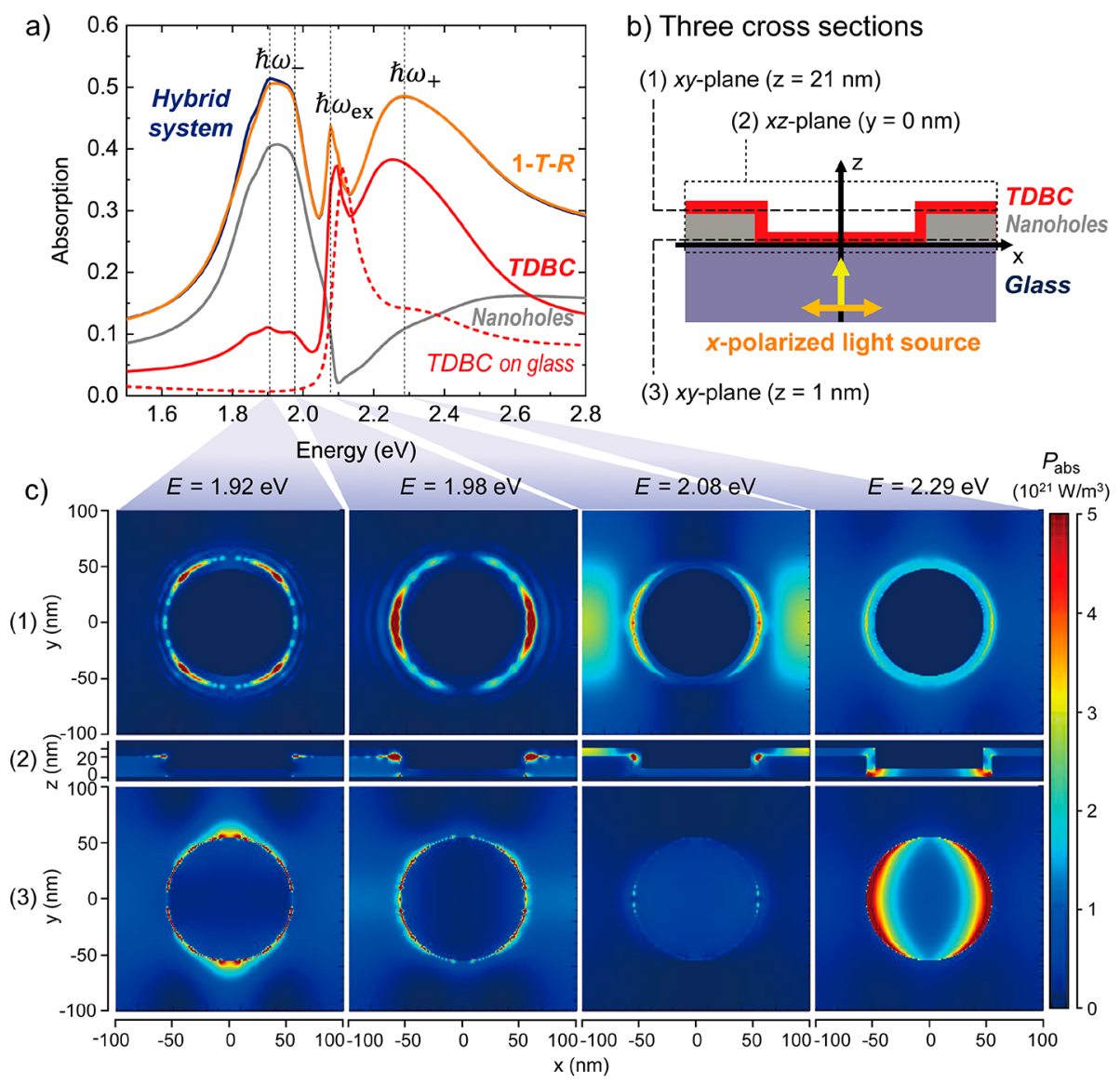

Figure 5. (a) Simulated power absorption by the individual materials: the TDBC layer (red), the silver nanohole film (gray), and their combination (blue). The simulated absorption spectrum for the hybrid system from the previous figure (orange) and a normalized simulated absorption spectrum for the bare TDBC layer on glass (red dashed) are included for comparison. (b) Schematic diagram of the nanohole structure used in the simulation: a $20 \mathrm{~nm}$ thick nanohole film on glass coated with TDBC. The locations of the three cross sections used in (c) are designated. Back illumination of the $x$-polarized light source from the glass side is illustrated by orange and yellow arrows, respectively indicating polarization and propagation direction. (c) Spatial distributions of the absorption at the resonance positions in each cross section described in (b).

different materials, which was not possible in the experiments (using BI, see the Supporting Information for details). This gives the separate absorption in the TDBC layer (red) and in the $20 \mathrm{~nm}$ thick nanohole film (gray), as presented in Figure 5a. The combination of those contributions (blue) matches the absorption spectra used in the previous figures (orange line), confirming the validity of this method. The most significant finding is that the Rabi splitting in absorption occurs both for the TDBC layer and for the nanohole film, which is a conclusive evidence of strong coupling. ${ }^{49}$ However, there are detailed features to be discussed further. Consistent with our discussion above, the peak near $\hbar \omega_{\mathrm{ex}}$ originates entirely from absorption by (nonhybridized) TDBC molecules. Furthermore, absorption by the TDBC layer dominates at $\hbar \omega_{+}$, while the nanohole film is responsible for a large fraction of the absorption at $\hbar \omega_{-}$. Part of this unbalanced absorption may be related to the asymmetric absorption of nonhybridized TDBC (red dashed line in Figure 5a), which can overemphasize the absorption by TDBC at $\hbar \omega_{+}$. Indeed, a more balanced absorption, in particular for the nanohole film, can be achieved by employing a symmetric Lorentzian model for the permittivity of TDBC in the simulations (see Figure S13). To obtain a more comprehensive understanding, we visualize $P_{\mathrm{abs}}$ at the different peak positions. Figure $5 \mathrm{~b}$ illustrates the locations of three selected cross sections, as used to display the two-dimensional $P_{\mathrm{abs}}$ intensity maps in Figure 5c. These three cross sections are (1) $x y$-plane slightly above the nanohole film, thereby intersecting only with TDBC; (2) xz-plane that passes through the center of the nanohole; and (3) $x y$-plane slightly above the bottom of the nanohole film. The results show that the absorption by the nanohole film near the $\hbar \omega_{-}$ peak includes a delocalized contribution throughout its entire metallic volume (two leftmost bottom columns of panels in Figure 5c). By contrast, the relatively small contribution from the TDBC layer in this range is rather localized to near the hole. At this lower range of excitation energies, absorption near the TDBC/nanohole interface showed dipolar (mid-left panels) to quadrupolar (left panels) mode transition. The multipolar mode can be ascribed to a mode hybridized with a higher order mode of the periodic nanohole array, ${ }^{36}$ which may, however, not be present for the experimental SRO nanohole films that only possess a single characteristic spacing. At $\hbar \omega_{\mathrm{ex}}$ (mid-right panels), light is primarily absorbed by the TDBC on top of the nanohole film. While some absorption results from the strong plasmonic field in the vicinity of the nanohole, the significant part originates from regions between holes, where one indeed can expect to find nonhybridized molecules. As excitation energy increases, the absorption by nonhybridized molecules first disappears, followed by the advent of another type of absorption near the $\hbar \omega_{+}$peak (right panels). Note that, although a large fraction of the absorption is concentrated inside the hole, a substantial fraction becomes 
delocalized between the holes, for both the TDBC coating and the metal film. These results indicate a partially delocalized nature of the hybridized polariton resonances, highlighting the possibilities of continuous plasmonic nanohole films and their use for applications such as influencing charge carrier transport in organic semiconductors. ${ }^{1,12}$

\section{CONCLUSIONS}

In summary, we report the first demonstration of strong plasmon-exciton coupling based on optically thin nanohole metasurfaces, here for scalable short-range ordered nanoholes coated with TDBC molecules. The absorptive resonances and suppressed transmission of these nanohole films enabled us to demonstrate vacuum Rabi splitting in both extinction and absorption. We find a linear dependence of vacuum Rabi splitting on the square root of TDBC concentration, which confirms that the system is in the strong coupling regime. Anticrossing analysis revealed different splitting in absorption and in extinction, with blue-shifted polariton extinction peaks attributed to Fano interference effects. This implies that absorption peaks form more intrinsic measures of the polariton resonances. Interestingly, the absorption spectra of the hybrid systems showed strong dependence on illumination direction, due to a combination of inherent asymmetry of the plasmonic nanohole metasurfaces and different light interaction with nonhybridized molecules. Besides opening up for new applications, such absorption directionality will be important to consider in future studies of coupling effects in asymmetric metasurfaces, not least in terms of illumination conditions for absorption measurements. All spectral features, including the directional behavior, were successfully reproduced by FDTD simulations, confirming the validity of our findings. Finally, strong coupling was further verified from the simulated spatial distribution of the local absorption in the hybrid system, which also showed indications of polaritons being delocalized between the nanoholes. Our study highlights the potential of employing optically thin plasmonic nanoholes as building blocks acting as strongly coupled plasmonic electrodes, with control over properties such as electrochemical reactivity ${ }^{10}$ and electrical conductivity. ${ }^{12}$

\section{ASSOCIATED CONTENT}

\section{S Supporting Information}

The Supporting Information is available free of charge on the ACS Publications website at DOI: 10.1021/acsphotonics.8b00679.

Materials and methods; radial distribution function of nanohole films; permittivity data of TDBC obtained by ellipsometry; peak extraction procedure; detailed discussion on Fano interference; line width estimation procedure; FDTD simulations using Lorentzian oscillators (PDF)

\section{AUTHOR INFORMATION}

\section{Corresponding Author}

*E-mail: magnus.jonsson@liu.se.

\section{ORCID $\odot$}

Timur Shegai: 0000-0002-4266-3721

Magnus P. Jonsson: 0000-0002-3002-3639

\section{Author Contributions}

M.P.J. proposed the initial idea and designed the project together with E.S.H.K. E.S.H.K. performed the measurements, simulations, and data analysis. S.C., N.A., and V.D. performed the ellipsometry experiments. S.S. and D.T. assisted in optical measurements and simulations. E.S.H.K., T.S., and M.P.J. wrote the manuscript through contributions of all authors. All authors have given approval to the final version of the manuscript.

\section{Notes}

The authors declare no competing financial interest.

\section{ACKNOWLEDGMENTS}

We acknowledge financial support from the Wenner-Gren Foundations, the Swedish Research Council, the Swedish Foundation for Strategic Research, the ÅForsk Foundation, the Royal Swedish Academy of Sciences, and the Swedish Government Strategic Research Area in Materials Science on Functional Materials at Linköping University (Faculty Grant SFO-Mat-LiU No. 2009 00971).

\section{REFERENCES}

(1) Ebbesen, T. W. Hybrid Light-Matter States in a Molecular and Material Science Perspective. Acc. Chem. Res. 2016, 49, 2403-2412.

(2) Baranov, D. G.; Wersäll, M.; Cuadra, J.; Antosiewicz, T. J.; Shegai, T. Novel Nanostructures and Materials for Strong LightMatter Interactions. ACS Photonics 2018, 5, 24-42.

(3) Törmä, P.; Barnes, W. L. Strong Coupling between Surface Plasmon Polaritons and Emitters: A Review. Rep. Prog. Phys. 2015, 78, 013901.

(4) Moilanen, A. J.; Hakala, T. K.; Törmä, P. Active Control of Surface Plasmon-Emitter Strong Coupling. ACS Photonics 2018, 5, 54-64.

(5) Wang, H.; Wang, H.-Y.; Cheng, Q.-D.; Xu, H.-L.; Sun, H.-B.; Huang, F.; Raja, W.; Toma, A.; Proietti Zaccaria, R. Hybrid-State Dynamics of Dye Molecules and Surface Plasmon Polaritons under Ultrastrong Coupling Regime. Laser Photon. Rev. 2018, 12, 1700176.

(6) Hofling, S.; Amthor, M.; Rahimi-Iman, A.; Kim, N. Y.; Fischer, J.; Savenko, I. G.; Kulakovski, V. D.; Shelykh, I. A.; Reitzenstein, S.; Forchel, A.; et al. An Electrically Pumped Polariton Laser. Nature 2015, 497, 348-352.

(7) Volz, T.; Reinhard, A.; Winger, M.; Badolato, A.; Hennessy, K. J.; Hu, E. L.; Imamoğlu, A. Ultrafast All-Optical Switching by Single Photons. Nat. Photonics 2012, 6, 605-609.

(8) Hutchison, J. A.; Schwartz, T.; Genet, C.; Devaux, E.; Ebbesen, T. W. Modifying Chemical Landscapes by Coupling to Vacuum Fields. Angew. Chem., Int. Ed. 2012, 51, 1592-1596.

(9) Canaguier-Durand, A.; Devaux, E.; George, J.; Pang, Y.; Hutchison, J. A.; Schwartz, T.; Genet, C.; Wilhelms, N.; Lehn, J. M.; Ebbesen, T. W. Thermodynamics of Molecules Strongly Coupled to the Vacuum Field. Angew. Chem., Int. Ed. 2013, 52, 10533-10536.

(10) Thomas, A.; George, J.; Shalabney, A.; Dryzhakov, M.; Varma, S. J.; Moran, J.; Chervy, T.; Zhong, X.; Devaux, E.; Genet, C.; et al. Ground-State Chemical Reactivity under Vibrational Coupling to the Vacuum Electromagnetic Field. Angew. Chem., Int. Ed. 2016, 55, 11462-11466.

(11) Munkhbat, B.; Wersäll, M.; Baranov, D. G.; Antosiewicz, T. J.; Shegai, T. Suppression of Photo-Oxidation of Organic Chromophores by Strong Coupling to Plasmonic Nanoantennas. Sci. Adv. 2018, 4, eaas9552.

(12) Orgiu, E.; George, J.; Hutchison, J. A.; Devaux, E.; Dayen, J. F.; Doudin, B.; Stellacci, F.; Genet, C.; Schachenmayer, J.; Genes, C.; et al. Conductivity in Organic Semiconductors Hybridized with the Vacuum Field. Nat. Mater. 2015, 14, 1123-1129. Hagenmüller, D. Cavity-Enhanced Transport of Charge. Phys. Rev. Lett. 2017, 119, 223601. 
(13) Feist, J.; Garcia-Vidal, F. J. Extraordinary Exciton Conductance Induced by Strong Coupling. Phys. Rev. Lett. 2015, 114, 196402.

(14) Chikkaraddy, R.; de Nijs, B.; Benz, F.; Barrow, S. J.; Scherman, O. A.; Rosta, E.; Demetriadou, A.; Fox, P.; Hess, O.; Baumberg, J. J. Single-Molecule Strong Coupling at Room Temperature in Plasmonic Nanocavities. Nature 2016, 535, 1-4.

(15) Bellessa, J.; Bonnand, C.; Plenet, J. C.; Mugnier, J. Strong Coupling between Surface Plasmons and Excitons in an Organic Semiconductor. Phys. Rev. Lett. 2004, 93, 036404.

(16) Hakala, T. K.; Toppari, J. J.; Kuzyk, A.; Pettersson, M.; Tikkanen, H.; Kunttu, H.; Torma, P. Vacuum Rabi Splitting and Strong-Coupling Dynamics for Surface-Plasmon Polaritons and Rhodamine 6G Molecules. Phys. Rev. Lett. 2009, 103, 053602.

(17) Gomez, D. E.; Vernon, K. C.; Mulvaney, P.; Davis, T. J. Surface Plasmon Mediated Strong Exciton - Photon Coupling in Semiconductor Nanocrystals. Nano Lett. 2010, 10, 274-278.

(18) Stete, F.; Koopman, W.; Bargheer, M. Signatures of Strong Coupling on Nanoparticles: Revealing Absorption Anticrossing by Tuning the Dielectric Environment. ACS Photonics 2017, 4, 16691676.

(19) Zengin, G.; Wersäll, M.; Nilsson, S.; Antosiewicz, T. J.; Käll, M.; Shegai, T. Realizing Strong Light-Matter Interactions between Single-Nanoparticle Plasmons and Molecular Excitons at Ambient Conditions. Phys. Rev. Lett. 2015, 114, 157401.

(20) Santhosh, K.; Bitton, O.; Chuntonov, L.; Haran, G. Vacuum Rabi Splitting in a Plasmonic Cavity at the Single Quantum Emitter Limit. Nat. Commun. 2016, 7, 11823.

(21) Rodriguez, S. R. K.; Rivas, J. G. Surface Lattice Resonances Strongly Coupled to Rhodamine 6G Excitons: Tuning the PlasmonExciton-Polariton Mass and Composition. Opt. Express 2013, 21, $1065-1070$.

(22) Väkeväinen, A. I.; Moerland, R. J.; Rekola, H. T.; Eskelinen, A. P.; Martikainen, J. P.; Kim, D. H.; Törmä, P. Plasmonic Surface Lattice Resonances at the Strong Coupling Regime. Nano Lett. 2014, 14, 1721-1727.

(23) Liu, W.; Lee, B.; Naylor, C. H.; Ee, H.; Park, J.; Johnson, A. T. C.; Agarwal, R. Strong Exciton - Plasmon Coupling in MoS 2 Coupled with Plasmonic Lattice. Nano Lett. 2016, 16, 1262-1269.

(24) Kravets, V. G.; Kabashin, A. V.; Barnes, W. L.; Grigorenko, A. N. Plasmonic Surface Lattice Resonances: A Review of Properties and Applications. Chem. Rev. 2018, 118, 5912-5951.

(25) Sannomiya, T.; Scholder, O.; Jefimovs, K.; Hafner, C.; Dahlin, A. B. Investigation of Plasmon Resonances in Metal Films with Nanohole Arrays for Biosensing Applications. Small 2011, 7, 16531663.

(26) Schwind, M.; Kasemo, B.; Zorić, I. Localized and Propagating Plasmons in Metal Films with Nanoholes. Nano Lett. 2013, 13, 17431750.

(27) Dahlin, A. B. Sensing Applications Based on Plasmonic Nanopores: The Hole Story. Analyst 2015, 140, 4748-4759.

(28) Eftekhari, F.; Escobedo, C.; Ferreira, J.; Duan, X.; Girotto, E. M.; Brolo, A. G.; Gordon, R.; Sinton, D. Nanoholes as Nanochannels: Flow-through Plasmonic Sensing. Anal. Chem. 2009, 81, 4308-4311.

(29) Jonsson, M. P.; Dahlin, A. B.; Feuz, L.; Petronis, S.; Höök, F. Locally Functionalized Short-Range Ordered Nanoplasmonic Pores for Bioanalytical Sensing. Anal. Chem. 2010, 82, 2087-2094.

(30) Barik, A.; Otto, L. M.; Yoo, D.; Jose, J.; Johnson, T. W.; Oh, S. H. Dielectrophoresis-Enhanced Plasmonic Sensing with Gold Nanohole Arrays. Nano Lett. 2014, 14, 2006-2012.

(31) Tordera, D.; Zhao, D.; Volkov, A. V.; Crispin, X.; Jonsson, M. P. Thermoplasmonic Semitransparent Nanohole Electrodes. Nano Lett. 2017, 17, 3145-3151.

(32) Ebbesen, T. W.; Lezec, H. J.; Ghaemi, H. F.; Thio, T.; Wolff, P.; Thio, T.; Wolff, P. Extraordinary Optical Transmission through Sub-Wavelength Hole Arrays. Nature 1998, 86, 1114-1117.

(33) Rodrigo, S. G.; de León-Pérez, F.; Martín-Moreno, L. Extraordinary Optical Transmission: Fundamentals and Applications. Proc. IEEE 2016, 104, 2288-2306.
(34) Braun, J.; Gompf, B.; Kobiela, G.; Dressel, M. How Holes Can Obscure the View: Suppressed Transmission through an Ultrathin Metal Film by a Subwavelength Hole Array. Phys. Rev. Lett. 2009, 103, 203901.

(35) Spevak, I. S.; Nikitin, A. Y.; Bezuglyi, E. V.; Levchenko, A.; Kats, A. V. Resonantly Suppressed Transmission and Anomalously Enhanced Light Absorption in Periodically Modulated Ultrathin Metal Films. Phys. Rev. B: Condens. Matter Mater. Phys. 2009, 79, 161406.

(36) Liu, M.; Song, Y.; Zhang, Y.; Wang, X.; Jin, C. Mode Evolution and Transmission Suppression in a Perforated Ultrathin Metallic Film with a Triangular Array of Holes. Plasmonics 2012, 7, 397-410.

(37) Rodrigo, S. G.; Martín-Moreno, L.; Nikitin, A. Y.; Kats, A. V.; Spevak, I. S.; García-Vidal, F. J. Extraordinary Optical Transmission through Hole Arrays in Optically Thin Metal Films. Opt. Lett. 2009, 34, 4-6.

(38) Luk'Yanchuk, B.; Zheludev, N. I.; Maier, S. A.; Halas, N. J.; Nordlander, P.; Giessen, H.; Chong, C. T. The Fano Resonance in Plasmonic Nanostructures and Metamaterials. Nat. Mater. 2010, 9, $707-715$.

(39) Dintinger, J.; Klein, S.; Bustos, F.; Barnes, W. L.; Ebbesen, T. W. Strong Coupling between Surface Plasmon-Polaritons and Organic Molecules in Subwavelength Hole Arrays. Phys. Rev. B: Condens. Matter Mater. Phys. 2005, 71, 035424.

(40) Wang, H.; Toma, A.; Wang, H.; Bozzola, A.; Miele, E.; Haddadpour, A.; Veronis, G.; De Angelis, F.; Wang, L.; Chen, Q.; et al. Role of Rabi Splitting Tuning in the Dynamics of Strongly Coupled J-Aggregates and Surface Plasmon Polaritons in Nanoholes Arrays. Nanoscale 2016, 8, 13445-13453.

(41) Wang, H.; Wang, H. Y.; Bozzola, A.; Toma, A.; Panaro, S.; Raja, W.; Alabastri, A.; Wang, L.; Chen, Q. D.; Xu, H. L.; et al. Dynamics of Strong Coupling between J-Aggregates and Surface Plasmon Polaritons in Subwavelength Hole Arrays. Adv. Funct. Mater. 2016, 26, $6198-6205$.

(42) Zanotto, S.; Tredicucci, A. Universal Lineshapes at the Crossover between Weak and Strong Critical Coupling in FanoResonant Coupled Oscillators. Sci. Rep. 2016, 6, 1-8.

(43) Jonsson, M. P.; Dahlin, A. B.; Jönsson, P.; Höök, F. Nanoplasmonic Biosensing with Focus on Short-Range Ordered Nanoholes in Thin Metal Films. Biointerphases 2008, 3, FD30-D40.

(44) Jackman, J. A.; Rahim Ferhan, A.; Cho, N.-J. Nanoplasmonic Sensors for Biointerfacial Science. Chem. Soc. Rev. 2017, 46, 36153660.

(45) Hanarp, P.; Sutherland, D.; Gold, J.; Kasemo, B. Nanostructured Model Biomaterial Surfaces Prepared by Colloidal Lithography. Nanostruct. Mater. 1999, 12, 429-432.

(46) Bellessa, J.; Symonds, C.; Vynck, K.; Lemaitre, A.; Brioude, A.; Beaur, L.; Plenet, J. C.; Viste, P.; Felbacq, D.; Cambril, E.; et al. Giant Rabi Splitting between Localized Mixed Plasmon-Exciton States in a Two-Dimensional Array of Nanosize Metallic Disks in an Organic Semiconductor. Phys. Rev. B: Condens. Matter Mater. Phys. 2009, 80, 033303.

(47) Melnikau, D.; Esteban, R.; Savateeva, D.; Sánchez-Iglesias, A.; Grzelczak, M.; Schmidt, M. K.; Liz-Marzán, L. M.; Aizpurua, J.; Rakovich, Y. P. Rabi Splitting in Photoluminescence Spectra of Hybrid Systems of Gold Nanorods and J-Aggregates. J. Phys. Chem. Lett. 2016, 7, 354-362.

(48) DeLacy, B. G.; Miller, O. D.; Hsu, C. W.; Zander, Z.; Lacey, S.; Yagloski, R.; Fountain, A. W.; Valdes, E.; Anquillare, E.; Soljačić, M.; et al. Coherent Plasmon-Exciton Coupling in Silver Platelet-JAggregate Nanocomposites. Nano Lett. 2015, 15, 2588-2593.

(49) Antosiewicz, T. J.; Apell, S. P.; Shegai, T. Plasmon-Exciton Interactions in a Core-Shell Geometry: From Enhanced Absorption to Strong Coupling. ACS Photonics 2014, 1, 454-463.

(50) Zengin, G.; Johansson, G.; Johansson, P.; Antosiewicz, T. J.; Käll, M.; Shegai, T. Approaching the Strong Coupling Limit in Single Plasmonic Nanorods Interacting with J-Aggregates. Sci. Rep. 2013, 3, 3074. 
(51) Zengin, G.; Gschneidtner, T.; Verre, R.; Shao, L.; Antosiewicz, T. J.; Moth-Poulsen, K.; Käll, M.; Shegai, T. Evaluating Conditions for Strong Coupling between Nanoparticle Plasmons and Organic Dyes Using Scattering and Absorption Spectroscopy. J. Phys. Chem. C 2016, 120, 20588-20596.

(52) Jönsson, G.; Tordera, D.; Pakizeh, T.; Jaysankar, M.; Miljkovic, V.; Tong, L.; Jonsson, M. P.; Dmitriev, A. Solar Transparent Radiators by Optical Nanoantennas. Nano Lett. 2017, 17, 6766-6772.

(53) Kosako, T.; Kadoya, Y.; Hofmann, H. F. Directional Control of Light by a Nano-Optical Yagi-Uda Antenna. Nat. Photonics 2010, 4, $312-315$.

(54) Shegai, T.; Chen, S.; Miljković, V. D.; Zengin, G.; Johansson, P.; Käll, M. A Bimetallic Nanoantenna for Directional Colour Routing. Nat. Commun. 2011, 2, 481.

(55) Wu, L.; Bai, P.; Zhou, X.; Li, E. P. Reflection and Transmission Modes in Nanohole-Array-Based Plasmonic Sensors. IEEE Photonics J. 2012, 4, 26-33.

(56) Rudin, S.; Reinecke, T. L. Oscillator Model for Vacuum Rabi Splitting in Microcavities. Phys. Rev. B: Condens. Matter Mater. Phys. 1999, 59, 10227-10233.

(57) Carretero-Palacios, S.; García-Vidal, F. J.; Martín-Moreno, L.; Rodrigo, S. G. Effect of Film Thickness and Dielectric Environment on Optical Transmission through Subwavelength Holes. Phys. Rev. B: Condens. Matter Mater. Phys. 2012, 85, 035417.

(58) Bortchagovsky, E. G.; Fischer, U. C. On the Modulation of Optical Transmission Spectra of Thin Dye Layers by a Supporting Medium. J. Chem. Phys. 2002, 117, 5384-5392.

(59) Schwartz, T.; Hutchison, J. A.; Genet, C.; Ebbesen, T. W. Reversible Switching of Ultrastrong Light-Molecule Coupling. Phys. Rev. Lett. 2011, 106, 196405.

(60) Genet, C.; Van Exter, M. P.; Woerdman, J. P. Fano-Type Interpretation of Red Shifts and Red Tails in Hole Array Transmission Spectra. Opt. Commun. 2003, 225, 331-336.

(61) Francescato, Y.; Giannini, V.; Maier, S. A. Plasmonic Systems Unveiled by Fano Resonances. ACS Nano 2012, 6, 1830-1838.

(62) Savasta, S.; Saija, R.; Ridolfo, A.; Di Stefano, O.; Denti, P.; Borghese, F. Nanopolaritons: Vacuum Rabi Splitting with a Single Quantum Dot in the Center of a Dimer Nanoantenna. ACS Nano 2010, 4, 6369-6376.

(63) Balci, S.; Kocabas, C.; Ates, S.; Karademir, E.; Salihoglu, O.; Aydinli, A. Tuning Surface Plasmon-Exciton Coupling via Thickness Dependent Plasmon Damping. Phys. Rev. B: Condens. Matter Mater. Phys. 2012, 86, 235402. 